\title{
Deriving the Jalilian-Marian-Iancu-McLerran-Weigert-Leonidov-Kovner equation with classical and quantum source terms
}

\author{
Kenji Fukushima ${ }^{1}$ \\ ${ }^{1}$ RIKEN BNL Research Center, Brookhaven National Laboratory, Upton, New York 11973-5000, USA
}

\begin{abstract}
We discuss the high-energy evolution equation in Quantum Chromodynamics (QCD) in the framework of the color glass condensate. We rewrite the generating functional of QCD with the color glass condensate into a representation with the density of states and a simple eikonal coupling. In this representation we introduce an auxiliary variable which is identified as the quantum color charge density consistent with non-commutativity in the operator formalism. We revisit the derivation of the Jalilian-Marian-Iancu-McLerran-Weigert-Leonidov-Kovner (JIMWLK) equation to clarify how the non-commutative nature among the color charge density operators can be disregarded in the regime where the gluon density is high.
\end{abstract}

PACS numbers: 12.38.-t,12.38.Aw

\section{INTRODUCTION}

There have been remarkable developments in our understanding of high-energy description of Quantum Chromodynamics (QCD) in the last decade. Although, in principle, main constituents of nuclei could be thoroughly governed by QCD dynamics, it would require enormous numerical computation to perform a first-principle calculation of QCD for the properties of nuclei. Most of problems encountered in QCD are non-perturbative, and besides, non-linear, meaning that gluons strongly interact with themselves.

Under some conditions feasible in experiments, however, one may hope to derive analytical information directly from QCD in a manageable way, which is not only of general interest in theoretical physics, but also of importance as an experimental testing ground for theoretical ideas in QCD. It is well known that the strong coupling constant, $\alpha_{s}$, is expected to be small for far energetic processes owing to asymptotic freedom, so that the perturbation theory would work. In fact, however, the perturbative expansion becomes reliable for processes in which the exchanged gluon carries a large momentum and it is possible that the momentum transfer remains small even for energetic processes. Moreover, even if the exchanged gluon conveys a sufficiently large momentum transfer, small $\alpha_{s}$ is not necessarily adequate to guarantee the validity of perturbative calculations.

Even when the perturbative expansion in terms of $\alpha_{s}$ seems plausible, resummation is needed whenever enhancing logarithms exist in the expansion series, which occurs in general for processes characterized by multiple (large) energy scales. This is actually the case for the processes we are discussing in this paper in which partons with small Bjorken-x are relevant. Then, two large scales $\Lambda_{\mathrm{QCD}} \ll t \ll s$ may give rise to a large logarithmic factor $\sim \ln [s / t] \sim \ln [1 / \mathrm{x}]$. Here Bjorken- $\mathrm{x}$ is intuitively interpreted as the longitudinal momentum fraction $\mathrm{x}=p^{+} / P^{+}$of a parton inside a target seen in the infinite momentum frame, $P^{+} \rightarrow \infty$; the target moves nearly at the speed of light in the positive $z$ direction, that is, along the $x^{+}=(z+t) / \sqrt{2}$ direction in the light-cone coordinate. Even though $\alpha_{s}$ is far smaller than unity, the perturbation theory in $\alpha_{s}$ breaks down if $\alpha_{s} \ln [1 / \mathrm{x}]$ turns out not to be small for $\mathrm{x} \ll 1$. In the leading-log approximation the resummation program with respect to such logarithmic factors has been developed, which brings about running in $\mathrm{x}$ for gluonic properties such as the gluon distribution. This is analogous to the famous $Q^{2}$ (momentum transfer) running of gauge coupling constant after the summation over leading $\ln \left[Q^{2} / \Lambda_{\mathrm{QCD}}^{2}\right]$ terms is taken in perturbative QCD. Insofar as only the perturbatively calculable part is concerned, it has been our common knowledge that such running in $\mathrm{x}$, or the small-x evolution, in the leading-log approximation obeys the Balitsky-Fadin-Kuraev-Lipatov (BFKL) equation 1, 2, 3, 4].

The BFKL equation lacks the saturation effects [5, 6, 7], however, that would be important physics when the parton (gluon) distribution is highly dense in the small-x region. Since the BFKL dynamics encompasses the evolution in $\mathrm{x}$ but not in $Q^{2}$, the transverse area is fixed and the number of partons increases in a fixed area as x goes smaller. Partons eventually overlap each other, indicating that non-linearity of QCD should be properly taken into account at a certain saturation scale $Q_{s}^{2}(\mathrm{x})$, and the parton number density must be saturated via recombination processes. The modern picture of this saturation mechanism is more suitably described by coherent gluon fields $\mathcal{A}_{a}^{\mu}$ representing small-x wee partons $\left[7,\left[8,[9]\right.\right.$. The larger-x partons are integrated out to lead to the gluon source $\rho^{a}$, from which the coherent fields $\mathcal{A}_{a}^{\mu}$ are created corresponding to smaller-x degrees of freedom. [This is a similar concept to the Weizsäcker-Williams approximation.] When $\mathcal{A}_{a}^{\mu}$ becomes as large as $\sim 1 / g$, non-linearity is crucial for the saturation. In the first approximation the source is, originally in literatures, just regarded as the color charge density carried by valence quarks inside the target hadron, supposing that the target is a large nucleus. Since quarks in different nucleons are uncorrelated, the color charge density can be considered as distributing randomly. The random distribution of 
strong gluon fields $\mathcal{A}_{a}^{\mu}$ is commonly referred to as the color glass condensate [10].

One can compute the small-x evolution, that is actually the renormalization group equation with the rapidity variable, from $\mathrm{x}$ down to $\mathrm{x}-d \mathrm{x}$ in the presence of the color glass condensate by assuming that one has such an effective theory of the color glass condensate at a certain $\mathrm{x}$ and one can retain the same framework at $\mathrm{x}-d \mathrm{x}$ after integrating out any degrees of freedom within the slice $d \mathrm{x}=d p^{+} / P^{+}$. The equation obtained in this way is to be considered as a natural extension of the BFKL equation with the saturation effect of dense gluons incorporated. There is an intrinsic energy scale $Q_{s}^{2}(\mathrm{x})$ which is large, so that the perturbative resummation becomes infraredly stable 11]. The theoretical framework has been well organized and the central result is now widely known as the Jalilian-Marian-Iancu-McLerran-Weigert-Leonidov-Kovner (JIMWLK) equation 10, 12, 13, 14, 15, 16, 17, 18].

The purpose of this paper is to present the derivation of the JIMWLK equation in a way different from the conventional arithmetic steps. The reasons why we shall attempt to rederive the well-established equation here are twofold: The author has recently proposed a representation for the gauge invariant source terms [19] which is equivalent with the form as used in literatures conventionally [12, 13, 14, 15, 16, 17, 18], but is rather suitable for treating the gauge invariance and non-commutative nature among color charge operators transparently. It would be a first-step check to confirm explicitly that such a representation proposed by the author really leads to the correct JIMWLK equation in the dense regime, which is necessary as a preparation for further applications. Another issue, that could provide a hint to approach the dilute regime where non-commutativity among color charge density operators is important, is that in our formalism we can clearly see under what approximation we have disregarded non-commutativity in the dense regime. The JIMWLK problem, i.e., the derivation of the evolution equation in the dense regime, has already been well understood itself and our final results shall not add any new materials to that. Nevertheless, looking at the derivation of the JIMWLK equation from another point of view as addressed in this paper should be useful to understand the JIMWLK dynamics more deeply and would give us a clue on the treatment beyond the approximations utilized in the dense regime. In short, the ideology of this paper is; the derivation is new, while the derived equation is already known.

In Sec. II we will quickly look over the philosophy of the color glass condensate and will articulate our final goal in advance, namely, the JIMWLK equation. We will present the central part of this paper in Sec. III where we will explain a step-by-step derivation of the JIMWLK equation along the formalism involving quantum color charge density. Our summary and conclusions are in Sec. IV Some discussions are followed in App. \ to account for connections between our derivation and the conventional one for the sake of more clarity.

\section{COLOR GLASS CONDENSATE}

We shall reiterate the physical situation we are considering here, though we have partially explained it. A nucleus (target) is moving fast toward the positive $z$ direction. In the light-cone coordinate the target is traveling along the temporal $x^{+}=(t+z) / \sqrt{2}$ direction while sitting on $x^{-}=(t-z) / \sqrt{2} \simeq 0$. In the momentum space language it bears a large longitudinal momentum $p^{+}=\left(p_{0}+p_{z}\right) / \sqrt{2} \sim \infty$. In the formulation of the color glass condensate the essential assumption is that there is a separation scale $\Lambda$ for $p^{+}$; all the fast partons with $\left|p^{+}\right|>\Lambda$ are integrated out to result in the classical color source $\bar{\rho}_{\Lambda}^{a}(\vec{x})$ which does not depend on $x^{+}$(i.e. static) because of the time dilatation relative to slower partons. The color orientation is assumed to distribute randomly at each spatial point $\vec{x}=\left(x^{-}, \boldsymbol{x}_{\perp}\right)$ with a weight $\mathcal{W}_{\Lambda}\left[\bar{\rho}_{\Lambda}^{a}\right]$. Under these assumptions the QCD generating functional is modeled as

$$
Z=\int \mathcal{D} \bar{\rho}_{\Lambda} \mathcal{W}_{\Lambda}\left[\bar{\rho}_{\Lambda}\right] \int^{\Lambda} \mathcal{D} A \delta\left[A^{+}\right] \exp \left\{i S_{\mathrm{YM}}[A]+i S_{W}\left[A^{-}, \bar{\rho}_{\Lambda}\right]\right\}
$$

It has been shown in 19 that the distribution $\bar{\rho}_{\Lambda}^{a}(\vec{x})$ is discrete at each spatial point at the microscopic level. In this paper, however, we shall work only in the dense regime so that we can approximate $\bar{\rho}_{\Lambda}^{a}(\vec{x})$ as a continuous function as treated conventionally. Here we have employed the light-cone gauge $\left(A^{+}=0\right)$ that enables us to cut-off the longitudinal momentum in a way free from a mixture of momenta carried by $A^{+}$through the covariant derivative $\partial^{+}-i g A^{+}$. It is quite important to note that $\bar{\rho}_{\Lambda}^{a}(\vec{x})$ has a support in the longitudinal direction as $0 \lesssim x^{-} \lesssim 1 / \Lambda$ as a result of the integration over fast gluons with an appropriate boundary condition imposed. This longitudinal structure is first recognized in [16, 17, 18] and can be also embedded in the weight functional $\mathcal{W}_{\Lambda}\left[\bar{\rho}_{\Lambda}\right]$ in a way leading to $\bar{\rho}_{\Lambda}^{a}(\vec{x})=0$ for $x^{-}>1 / \Lambda$ [20]. The JIMWLK equation is, as we will see later, consistent with the support structure as it should be; along with the quantum evolution in $\mathcal{W}_{\Lambda}\left[\bar{\rho}_{\Lambda}\right]$ the edge of the support diffuses to larger $x^{-}$. In our notation we do not have to attach the scale to the source specifically once $\mathcal{W}_{\Lambda}\left[\bar{\rho}_{\Lambda}\right]$ accommodates the support structure , but we will stick to denoting $\bar{\rho}_{\Lambda}^{a}(\vec{x})$ in this paper to remind us of the longitudinal structure.

The support in $x^{-}$is intuitively to be interpreted as a coincidence of the momentum rapidity and the space-time 
rapidity of fast partons as argued in [16]. That is, the rapidity variable,

$$
\tau=\ln \left[\frac{1}{\mathrm{x}}\right]
$$

is defined as a momentum rapidity difference between the target and the parton. If the particle moves along the longitudinal direction and its velocity is not changed, which is actually the case in the eikonal approximation, the momentum rapidity is identical to the space-time rapidity. Therefore, if the target is at the position of $x_{0}^{-}$, then the parton with the rapidity difference $\tau$ is located at $x_{\tau}^{-}=x_{0}^{-} e^{\tau}$. Since $\bar{\rho}_{\Lambda}^{a}(\vec{x})$ has an origin from fast gluons with $\tau<\ln \left[P^{+} / \Lambda\right]$ in the color glass condensate picture, its distribution is within $x_{0}^{-} \simeq 0 \lesssim x^{-} \lesssim 1 / \Lambda \simeq x_{\tau}^{-}$. Hereafter we will often make use of $\tau$ to mean a reference rapidity $\tau=\ln \left[P^{+} / \Lambda\right]$ instead of using the separation scale $\Lambda$ like $\mathcal{W}_{\tau}\left[\bar{\rho}_{\tau}\right]$ signifying $\mathcal{W}_{\Lambda}\left[\bar{\rho}_{\Lambda}\right]$ equivalently.

In this paper we expressly call $\bar{\rho}_{\Lambda}^{a}(\vec{x})$ the classical color charge density to distinguish it from the quantum one that fulfills the covariant conservation and the canonical commutation relations at the operator level. [We will explain what this means exactly in the next section.] In short $\bar{\rho}_{\Lambda}^{a}(\vec{x})$ turns out to be not a source for quantum fields but a distribution of fast partons, which is a well-defined concept at the classical level. The recognition of $\bar{\rho}_{\Lambda}^{a}(\vec{x})$ as a classical quantity characterizing the particle distribution has been discussed already in [16, 17, 18] where our $\bar{\rho}_{\Lambda}^{a}(\vec{x})$ is denoted by $\nu$ in order to distinguish it from the genuine source for quantum fluctuations. Its significance has also been emphasized recently in 19 .

The source action $S_{W}\left[A^{-}, \bar{\rho}_{\Lambda}\right]$ in (11) is required to be gauge invariant and to be close to the simple eikonal coupling $\sim \operatorname{tr}\left\{\bar{\rho} A^{-}\right\}$not to affect the equations of motion. In the most conventional formulation the source terms have been anticipated as

$$
i S_{W}\left[A^{-}, \bar{\rho}_{\Lambda}\right]=-\frac{1}{g N_{c}} \int d^{3} x \operatorname{tr}\left\{\bar{\rho}_{\Lambda}(\vec{x}) W\left[A^{-}\right](\vec{x})\right\} .
$$

with the Wilson line along the light-cone temporal direction,

$$
W\left[A^{-}\right](\vec{x})=\mathcal{P} \exp \left[i g \int_{-\infty}^{\infty} d x^{+} A_{a}^{-}\left(x^{+}, \vec{x}\right) T^{a}\right],
$$

defined in the color adjoint representation. Here the source matrix is defined in the adjoint basis; $\bar{\rho}_{\Lambda}=\bar{\rho}_{\Lambda}^{a} T^{a}$. As usual $\mathcal{P}$ stands for time (i.e. $x^{+}$) ordering. The choice of $S_{W}\left[A^{-}, \bar{\rho}_{\Lambda}\right]$, however, is not unique and there are as many variants as one likes that satisfy gauge invariance and lead to the same equations of motion. Recently it has been revealed in [19] that a careful treatment of the fast gluon integration gives rise to a logarithmic form,

$$
i S_{W}\left[A^{-}, \bar{\rho}_{\Lambda}\right]=-\frac{1}{g N_{c}} \int d^{3} x \operatorname{tr}\left\{\bar{\rho}_{\Lambda}(\vec{x}) \ln W\left[A^{-}\right](\vec{x})\right\},
$$

which was proposed first in 21] motivated by Wong's equation. We shall adopt the latter [5] rather than the conventional choice (3) throughout this paper when the explicit form is necessary, though this choice should be irrelevant to the final results at least within the scope of the JIMWLK problem.

Now let us see what follows from the generating functional (11). The classical picture of the color glass condensate results from the tree-level approximation of (11). The expectation value of an operator of gauge fields, $\langle\mathcal{O}[A]\rangle_{\Lambda}$, is evaluated via $\langle\langle\mathcal{O}[A]\rangle\rangle_{\bar{\rho}_{\Lambda}}$ defined as

$$
\langle\langle\mathcal{O}[A]\rangle\rangle_{\bar{\rho}_{\Lambda}}=\frac{1}{z_{\Lambda}} \int^{\Lambda} \mathcal{D} A \delta\left[A^{+}\right] \mathcal{O}[A] e^{i S_{\mathrm{YM}}[A]+i S_{W}\left[A^{-}, \bar{\rho}_{\Lambda}\right]}
$$

with the generating functional $z_{\Lambda}\left[\bar{\rho}_{\Lambda}\right]=\int{ }^{\Lambda} \mathcal{D} A \delta\left[A^{+}\right] e^{i S_{\mathrm{YM}}+i S_{W}}$ for states with a given $\bar{\rho}_{\Lambda}$. Then one can estimate $\langle\mathcal{O}[A]\rangle_{\Lambda}$ as an average over the $\bar{\rho}_{\Lambda}$-distribution and make a classical approximation;

$$
\langle\mathcal{O}[A]\rangle_{\Lambda}=\int D \bar{\rho}_{\Lambda} \mathcal{W}_{\Lambda}\left[\bar{\rho}_{\Lambda}\right]\langle\langle\mathcal{O}[A]\rangle\rangle_{\bar{\rho}_{\Lambda}} \simeq \int \mathcal{D} \bar{\rho}_{\Lambda} \mathcal{W}_{\Lambda}\left[\bar{\rho}_{\Lambda}\right] \mathcal{O}\left[\mathcal{A}\left[\bar{\rho}_{\Lambda}\right]\right]
$$

using the solution of the Yang-Mills equations of motion $\mathcal{A}_{a}^{\mu}(x)$ as a function of the source,

$$
\left.\frac{\delta S_{\mathrm{YM}}[A]}{\delta A_{a}^{\mu}(x)}\right|_{\mathcal{A}}+\left.\frac{\delta S_{W}\left[A^{-}, \bar{\rho}_{\Lambda}\right]}{\delta A_{a}^{\mu}(x)}\right|_{\mathcal{A}}=0
$$


This is in fact a stationary-point approximation and expected to be legitimate when $\mathcal{W}_{\Lambda}\left[\bar{\rho}_{\Lambda}\right]$ allows for predominant contributions from large $\bar{\rho}_{\Lambda} \sim 1 / g$ and thus large $\mathcal{A}_{a}^{\mu}\left[\bar{\rho}_{\Lambda}\right]$ in the saturation regime. Here it is understood that the weight functional is properly normalized; $\int \mathcal{D} \bar{\rho}_{\Lambda} \mathcal{W}_{\Lambda}\left[\bar{\rho}_{\Lambda}\right]=1$. Since $\bar{\rho}_{\Lambda}$ is just an integration variable in (77), the $\Lambda$-dependence in the functional form of $\mathcal{W}_{\Lambda}$ solely governs the evolution of the quantity $\langle\mathcal{O}[A]\rangle_{\Lambda}$ as $\Lambda$ changes. This evolution is what the JIMWLK equation describes.

The explicit classical solution to the QCD equations of motion, $D_{\nu} F^{\nu \mu}-\delta^{\mu+} \bar{\rho}_{\Lambda}=0$ (with an ansatz $\mathcal{A}^{-}=0$ which makes the source term simple), is given in great details in literatures [10]. The covariant gauge $\left(\partial_{\mu} \widetilde{A}^{\mu}=0\right.$ where we follow the convention that covariant gauge quantities are specifically denoted with a tilde) has a one-component solution which is as simple as

$$
\widetilde{\mathcal{A}}^{\mu}\left[\bar{\rho}_{\Lambda}\right]=\delta^{\mu+} \alpha_{\Lambda}^{a}(\vec{x}) T^{a},
$$

where $\alpha_{\Lambda}^{a}(\vec{x})$ is a solution of the Poisson equation in the two-dimensional transverse plane, that is,

$$
\alpha_{\Lambda}^{a}(\vec{x})=-\int d^{2} \boldsymbol{y}_{\perp}\left\langle\boldsymbol{x}_{\perp}\left|\frac{1}{\boldsymbol{\partial}_{\perp}^{2}}\right| \boldsymbol{y}_{\perp}\right\rangle \bar{\rho}_{\Lambda}^{a}\left(x^{-}, \boldsymbol{y}_{\perp}\right)=\int \frac{d^{2} \boldsymbol{y}_{\perp}}{4 \pi} \ln \frac{1}{\left|\boldsymbol{x}_{\perp}-\boldsymbol{y}_{\perp}\right|^{2} \mu^{2}} \bar{\rho}_{\Lambda}^{a}\left(x^{-}, \boldsymbol{y}_{\perp}\right)
$$

Here $\mu^{2}$ is an infrared regulator that eventually cancels in the calculation for gauge invariant objects. The cancellation of $\mu^{2}$ naturally occurs; $1 / \mu$ gives a scale of long-range force from the source $\bar{\rho}_{\Lambda}$. The force extends to infinity causing an infrared singularity at $\mu \rightarrow 0$. If an object is color singlet as a whole and its finer structure inside $\bar{\rho}_{\Lambda}^{a}(\vec{x})$ is not resolved from a distance, there should be no color force any longer at distance and thus no infrared singularity.

The solution in the light-cone gauge $\left(A^{+}=0\right)$, which is necessary in the calculation of quantum fluctuations, is available by the gauge rotation $\widetilde{\mathcal{A}}^{\mu} \rightarrow V\left(\widetilde{\mathcal{A}}^{\mu}-\partial^{\mu} / i g\right) V^{\dagger}$ eliminating $\widetilde{\mathcal{A}}^{+}$, that results in

$$
\mathcal{A}_{a}^{\mu}\left[\bar{\rho}_{\Lambda}\right]=-\delta^{\mu i} \frac{1}{i g} V_{x^{-},-\infty}\left(\boldsymbol{x}_{\perp}\right) \partial^{i} V_{x^{-},-\infty}^{\dagger}\left(\boldsymbol{x}_{\perp}\right)
$$

with the gauge rotation matrix

$$
V_{x^{-},-\infty}^{\dagger}\left(\boldsymbol{x}_{\perp}\right)=\mathcal{P} \exp \left[i g \int_{-\infty}^{x^{-}} d z^{-} \alpha_{\Lambda}^{a}\left(z^{-}, \boldsymbol{x}_{\perp}\right) T^{a}\right] .
$$

Here $\mathcal{P}$ represents the ordering in $x^{-}$unlike $x^{+}$-ordering in (4). In this paper, in order to avoid tangled notations like $\widetilde{\bar{\rho}}_{\Lambda}$, we would use only $\bar{\rho}_{\Lambda}$ with a remark about the gauge choice if necessary. In the above discussion $\bar{\rho}_{\Lambda}$ is all in the covariant gauge, while the light-cone gauge solution (11) satisfies the equations of motion for the source in the light-cone gauge, namely, $V_{x^{-},-\infty} \bar{\rho}_{\Lambda} V_{x^{-},-\infty}^{\dagger}$ written in terms of the covariant gauge source.

From these relations we can choose to use $\alpha_{\Lambda}^{a}(\vec{x})$ to express a function of $\bar{\rho}_{\Lambda}^{a}(\vec{x})$. Since $\bar{\rho}_{\Lambda}^{a}(\vec{x})$ has the support over $0 \lesssim x^{-} \lesssim 1 / \Lambda$, so does $\alpha_{\Lambda}^{a}(\vec{x})$. One might seem perplexed at this at first glance; since the $x^{-}$-structure is such localized, $\alpha_{\Lambda}^{a}(\vec{x})$ carries a large longitudinal momentum $\left|p^{+}\right|>\Lambda$ like fast partons generating $\bar{\rho}_{\Lambda}(\vec{x})$. At the same time the classical fields should describe wee partons with $\left|p^{+}\right|<\Lambda$. This is not a contradiction, however. The point is that the scale separation in $p^{+}$, as we have mentioned, makes sense not in the covariant gauge but in the light-cone gauge only. Actually $\mathcal{A}^{i}\left[\bar{\rho}_{\Lambda}\right]$ in the light-cone gauge is delocalized and takes a nonvanishing value at $x^{-} \gtrsim 1 / \Lambda$. [It should be noted that the retarded boundary condition is implicit in the choice of (12) so that only positive $x^{-}$is taken.] Recalling a coincidence between the momentum and space-time rapidities, the light-cone gauge solution $\mathcal{A}^{i}\left[\bar{\rho}_{\Lambda}\right]$ distributing over $x^{-} \gtrsim 1 / \Lambda$ surely corresponds to slow partons.

We are now ready to write the JIMWLK equation down in a well-known form in terms of $\alpha_{\tau}^{a}(\vec{x})$ using the rapidity variable $\tau$ defined in (2);

$$
\frac{\partial}{\partial \tau} \mathcal{W}_{\tau}\left[\alpha_{\tau}\right]=\frac{1}{2} \int d^{2} \boldsymbol{x}_{\perp} d^{2} \boldsymbol{y}_{\perp} \frac{\delta}{\delta \alpha_{\tau}^{a}\left(x_{\tau}^{-}, \boldsymbol{x}_{\perp}\right)} \eta^{a b}\left(\boldsymbol{x}_{\perp}, \boldsymbol{y}_{\perp}\right) \frac{\delta}{\delta \alpha_{\tau}^{b}\left(x_{\tau}^{-}, \boldsymbol{y}_{\perp}\right)} \mathcal{W}_{\tau}\left[\alpha_{\tau}\right],
$$

where the derivatives act only on the edge of the support $x^{-}=x_{\tau}^{-}$and

$$
\eta^{a b}\left(\boldsymbol{x}_{\perp}, \boldsymbol{y}_{\perp}\right)=\frac{1}{\pi} \int \frac{d^{2} \boldsymbol{z}_{\perp}}{(2 \pi)^{2}} \mathcal{K}\left(\boldsymbol{x}_{\perp}, \boldsymbol{y}_{\perp}, \boldsymbol{z}_{\perp}\right)\left[1-V^{\dagger}\left(\boldsymbol{z}_{\perp}\right) V\left(\boldsymbol{y}_{\perp}\right)-V^{\dagger}\left(\boldsymbol{x}_{\perp}\right) V\left(\boldsymbol{z}_{\perp}\right)+V^{\dagger}\left(\boldsymbol{x}_{\perp}\right) V\left(\boldsymbol{y}_{\perp}\right)\right]^{a b}
$$

with the transverse kernel,

$$
\mathcal{K}\left(\boldsymbol{x}_{\perp}, \boldsymbol{y}_{\perp}, \boldsymbol{z}_{\perp}\right)=(2 \pi)^{2}\left\langle\boldsymbol{z}_{\perp}\left|\frac{\boldsymbol{\partial}_{\perp}}{\boldsymbol{\partial}_{\perp}^{2}}\right| \boldsymbol{x}_{\perp}\right\rangle \cdot\left\langle\boldsymbol{z}_{\perp}\left|\frac{\boldsymbol{\partial}_{\perp}}{\boldsymbol{\partial}_{\perp}^{2}}\right| \boldsymbol{y}_{\perp}\right\rangle=\frac{\left(\boldsymbol{x}_{\perp}-\boldsymbol{z}_{\perp}\right) \cdot\left(\boldsymbol{y}_{\perp}-\boldsymbol{z}_{\perp}\right)}{\left(\boldsymbol{x}_{\perp}-\boldsymbol{z}_{\perp}\right)^{2}\left(\boldsymbol{y}_{\perp}-\boldsymbol{z}_{\perp}\right)^{2}} .
$$


Here $V^{\dagger}\left(\boldsymbol{x}_{\perp}\right)=V_{x_{\tau}^{-},-\infty}^{\dagger}\left(\boldsymbol{x}_{\perp}\right) \simeq V_{+\infty,-\infty}^{\dagger}\left(\boldsymbol{x}_{\perp}\right)$ with the definition (12).

The derivation of the JIMWLK equation (13) is our goal pursued in this paper. Usually one derives the JIMWLK equation from the fact that the generating functional (1) is independent of the separation scale $\Lambda$ as we will explain in some details in App. W We shall here take a different starting point: The important observation pointed out in [19] is that the generating functional is written equivalently in a different form with an auxiliary variable $\rho$ as

$$
Z=\int \mathcal{D} \rho \mathcal{N}_{\Lambda}[\rho] \int^{\Lambda} \mathcal{D} A \delta\left[A^{+}\right] \exp \left[i S_{\mathrm{YM}}[A]-i \int d^{4} x \rho^{a}(x) A_{a}^{-}(x)\right]
$$

with the density of states $\mathcal{N}_{\Lambda}[\rho]$ defined as

$$
\mathcal{N}_{\Lambda}[\rho]=\int \mathcal{D} \bar{\rho}_{\Lambda} \mathcal{W}_{\Lambda}\left[\bar{\rho}_{\Lambda}\right] \exp \left[-\frac{1}{g N_{c}} \int d^{3} x \operatorname{tr}\left\{\bar{\rho}_{\Lambda}(\vec{x}) \ln W[-i \delta / \delta \rho](\vec{x})\right\}\right] \delta[\rho] .
$$

One can immediately prove that (16) returns exactly to (11) after the $\rho$-integration. Because (16) is mathematically equivalent with (1), one should be able to derive the same evolution equation from the $\Lambda$-independence of $Z$ as written in (16). We are attempting to make sure this expectation in what follows. Let us see as an instant check how the equivalent classical picture of the color glass condensate arises. This is simply concluded from a claim that $\rho^{a}(x)$ is static, which corresponds to an approximation that non-commutativity is disregarded as we will argue later (see discussions around (35)). There is then no discrimination between $\bar{\rho}_{\Lambda}^{a}(\vec{x})$ and $\rho^{a}(x)$ and moreover $\mathcal{N}_{\Lambda}[\rho]$ is reduced to $\mathcal{W}_{\Lambda}\left[\bar{\rho}_{\Lambda}\right]$ and two representations become trivially identical. The quantum evolution is, however, not so trivial. The derivation of the JIMWLK equation based on (16) is the main issue of this paper and elucidated in Sec. III]

\section{DERIVATION WITH QUANTUM COLOR CHARGE DENSITY}

We will derive the JIMWLK equation (13) with our emphasis on the quantum nature of color charge operators. We would remark here again that the derivation presented in this section is our central achievement of this paper. First in Sec. III we will explain about non-commutativity among quantum color charge density from the field theoretical point of view, and then we will make proceed in the concrete calculus in Sec. IIIB

\section{A. Quantum color charge density}

Before addressing the computational steps, let us clarify the meaning of quantum color charge density and explain why we are interested in that. In the canonical quantization, in gauge theories, the color charge density operators obey the commutation relation at equal time,

$$
\left[\hat{\rho}^{a}\left(x^{+}, \vec{x}\right), \hat{\rho}^{b}\left(x^{+}, \vec{y}\right)\right]=-i g f^{a b c} \hat{\rho}^{c}\left(x^{+}, \vec{x}\right) \delta^{(3)}(\vec{x}-\vec{y}),
$$

which one can easily confirm by using the canonical quantization conditions. The commutation relation (18) holds for the source of any (fermionic or bosonic) matter field. In the operator formalism of quantization, (18) is necessary for consistency between Gauss' law and gauge invariance, that is, the secondary constraint in the nonabelian gauge theory (i.e. Gauss' law) is first-class if we use Dirac's nomenclature for quantization of singular systems [22]. If the theory is formulated in the functional integral formalism, (18) is expressed by the expectation values in terms of classical fields. In the functional integral formalism, by construction, the expectation value is taken in a time-ordered way and (18) is reexpressed as

$$
\left.\left\langle\mathcal{O}[\rho]\left\{\rho^{a}\left(x^{+}, \vec{x}\right) \rho^{b}\left(x^{+}-\eta, \vec{y}\right)-\rho^{b}\left(x^{+}, \vec{y}\right) \rho^{a}\left(x^{+}-\eta, \vec{x}\right)\right\}\right\rangle\right|_{\eta \rightarrow 0^{+}}=-i g f^{a b c}\left\langle\mathcal{O}[\rho] \rho^{c}\left(x^{+}, \vec{x}\right)\right\rangle \delta^{(3)}(\vec{x}-\vec{y})
$$

for arbitrary functionals $\mathcal{O}[\rho]$.

In the JIMWLK problem relevant in the dense regime where the color charge density is as large as $\sim 1 / g$, we can ignore the right-hand side of (19). Then, non-commutativity does not play any significant role in the JIMWLK evolution equation (apart from generating non-local vertices corresponding to the expansion of the source terms [23]). It is a challenging problem to transcend the JIMWLK equation towards the dilute regime where (18) or (19) must be taken into account. This attempt has been in part successful so far; the expansion in terms of the charge density derivative has been improved up to the forth order [24] and now the general Hamiltonian for all orders has been known [25, 26], while the complete quantization procedure has not been quite clear yet. In the dilute regime the quantum 
evolution has also been studied in the color dipole picture [27] which can be connected to the color glass formalism [28].

In the operator formalism the Heisenberg equation with (18) describes the evolution, given the Hamiltonian is known as a function of the charge density operators. Since the color glass formulation, as we have seen in Sec. [II is naturally understood in the form of the functional integral, it is desired to formulate the evolution equation consistent with (19) within the functional integral description. Recalling that the color charge operators can be regarded as spin operators aligned in color group space, one can anticipate a functional integral representation from knowledge on the spin system. That is, the action for the spin system is known to consist of the dynamical and geometrical parts. In the language of quantum mechanics, the action is the integral of the Lagrangian which is given as $L(q)=p \dot{q}-H(p)$ with a variable $q$ and its canonical conjugate $p$. The dynamical and geometrical parts correspond to the integral of $H(p)$ and $p \dot{q}$ respectively. In fact the geometrical part has an interpretation as the topological phase which is often referred to as Berry's phase. Therefore, some attempts to augment the formalism with Berry's phase in color space have been proposed in order to handle (19) properly [23, 25].

Recently, in [19], a different way to deal with non-commutativity (19) has been proposed in the functional integral formulation. The advantage of that proposed method as compared with the representation with Berry's phase is that the necessary modification to the conventional formulation is minimal. There is no need to add Berry's phase into the formulation. The point is that $\rho^{a}(x)$ appearing in (16) rather than $\bar{\rho}_{\Lambda}^{a}(\vec{x})$ fulfills (19) and can be regarded as quantum color charge density. That is simply because the color charge density operator corresponds to $i \delta / \delta A_{a}^{-}(x)$ acting on the source action $\exp \left[i S_{W}\left[A^{-}, \rho\right]\right]$. If we choose the source action as in (16), then $i \delta / \delta A_{a}^{-}(x)$ is replaced by $\rho^{a}(x)$ coming from the eikonal coupling. It is obvious at this point that non-commutativity is lost if the $x^{+}$-dependence of $\rho^{a}(x)$ is dropped. In the next subsection we will see how this $x^{+}$-dependence is eventually abandoned in deriving the JIMWLK equation. This means, in other words, that one would be able to surpass the JIMWLK equation if one keeps the full $x^{+}$-dependence, which is beyond the scope of this present paper.

\section{B. Renormalization group equation}

We shall elaborate the renormalization group equation here from the fact that the density of states $\mathcal{N}_{\Lambda}[\rho]$ evolves so as to keep the whole generating functional $Z$ independent of the separation scale $\Lambda$. That is, $Z$ given in (16) can be written at a different separation scale $b \Lambda$ for arbitrary $b$. In this paper $b$ is assumed to be small as usual, i.e. $b \ll 1$ and we are working up to the leading order of $\alpha_{s} \ln [1 / b] \sim \alpha_{s} d \tau$ and will finally take the limit of $b \rightarrow 1$ to acquire the differential equation in $\tau$. Then one will reach $\mathcal{N}_{b \Lambda}[\rho]$ expressed in terms of $\mathcal{N}_{\Lambda}[\rho]$ accompanied by contributions from the integration over degrees of freedom inside the scale from $\Lambda$ to $b \Lambda$. Such a relation is nothing but the renormalization group equation for $\mathcal{N}_{\Lambda}[\rho]$ that flows according to the scale $\Lambda$ or the rapidity $\tau$.

Let us briefly summarize in advance each step for the calculation procedure for the sake of guiding what will transpire below.

(Step-1) We will first start with the generating functional $Z$ written at the separation scale $\Lambda$. We will decompose the gauge fields into the slow and fast parts according to the longitudinal momentum $p^{+}$, and we will expand the Yang-Mills action up to the quadratic order in semi-fast gluons $a_{a}^{\mu}$ which we will define there.

(Step-2) We will perform the Gaussian integration with respect to $a_{a}^{\mu}$ and will reach an expression of the generating functional $Z$ with the gauge field fluctuation below the scale $b \Lambda$.

(Step-3) We will equate the counterpart of the density of states with $\mathcal{N}_{b \Lambda}[\rho]$ after the Gaussian integration. In the limit of $b \rightarrow 1$ we will acquire the differential equation, that is, the renormalization group equation.

(Step-4) We will rewrite the renormalization group equation in terms of $\mathcal{W}_{\Lambda}\left[\bar{\rho}_{\Lambda}\right]$.

In the rest of this subsection we shall closely explain each step in order. In App. @ for comparison, we will sketch the conventional derivation of the JIMWLK equation directly for $\mathcal{W}_{\Lambda}\left[\bar{\rho}_{\Lambda}\right]$ in accord with (Step-1), (Step-2), and (Step-3) above.

(Step-1) In order to eliminate degrees of freedom within the momentum shell to be integrated, we shall decompose the gauge fields into the semi-fast gluons $a_{a}^{\mu}$ whose longitudinal momenta lie in the strip, $b \Lambda<\left|p^{+}\right|<\Lambda$, the classical solution, $\mathcal{A}_{a}^{\mu}\left[\bar{\rho}_{b \Lambda}\right]$ of (11), and the rest $\delta A_{a}^{\mu}$ with $\left|p^{+}\right|<b \Lambda$, that is,

$$
A_{a}^{\mu} \rightarrow a_{a}^{\mu}+\delta^{\mu i} \mathcal{A}_{a}^{i}\left[\bar{\rho}_{b \Lambda}\right]+\delta^{\mu-} \delta A_{a}^{-} .
$$

We assume that the transverse fluctuation around $\mathcal{A}_{a}^{i}$ is small enough to be negligible. Actually one can show that the only current (defined as the conjugate to $\delta A_{a}^{\mu}$ ) correlators to be retained in the leading-log approximation are correlators of the charge density (conjugate to $\delta A_{a}^{-}$) [16]. Therefore the specific decomposition (20) is validated under 
the present approximation. It should be noted that from the argument below (12) we know that the classical solution appears as a result of the stationary point approximation in evaluating the generating functional, and thus the classical part in the above decomposition (20) should be that for $\bar{\rho}_{b \Lambda}$ (not for $\bar{\rho}_{\Lambda}$ ). This is because $a_{a}^{\mu}$ is integrated out and $\mathcal{A}_{a}^{i}\left[\bar{\rho}_{b \Lambda}\right]$ should correspond to the stationary point of fields which have $\left|p^{+}\right|<b \Lambda$.

Then, it is straightforward to expand the Yang-Mills action in the generating functional at the scale $\Lambda$ in terms of $a_{a}^{\mu}$ up to the quadratic order.

$$
\begin{aligned}
& Z \simeq \int \mathcal{D} \rho \mathcal{N}_{\Lambda}[\rho] \int^{b \Lambda} \mathcal{D} \delta A \delta\left[\delta A^{+}\right] \exp \left[i S_{\mathrm{YM}}\left[\mathcal{A}\left[\bar{\rho}_{b \Lambda}\right]+\delta A\right]-i \int d^{4} x \rho^{a}(x) \delta A_{a}^{-}(x)\right] \\
& \times \int_{b \Lambda}^{\Lambda} \mathcal{D} a \delta\left[a^{+}\right] \exp \left[i \int d^{4} x \frac{\delta S_{\mathrm{YM}}}{\delta A_{a}^{\mu}(x)} a_{a}^{\mu}(x)+\frac{i}{2} \int d^{4} x d^{4} y \frac{\delta^{2} S_{\mathrm{YM}}}{\delta A_{a}^{\mu}(x) \delta A_{b}^{\nu}(y)} a_{a}^{\mu}(x) a_{b}^{\nu}(y)-i \int d^{4} x \rho^{a}(x) a_{a}^{-}(x)\right]
\end{aligned}
$$

where the derivatives of the Yang-Mills action are taken at $\mathcal{A}_{a}^{i}\left[\bar{\rho}_{b \Lambda}\right]+\delta A_{a}^{-}$.

(Step-2) Since (21) is quadratic in $a_{a}^{\mu}$ one can easily perform the Gaussian integration to have

$$
\begin{gathered}
\int_{b \Lambda}^{\Lambda} \mathcal{D} a \delta\left[a^{+}\right] \exp \left[-\frac{1}{2} \int d^{4} x d^{4} y\left\{a_{a}^{\mu}(x)-i \int d^{4} z\left(\frac{\delta S_{\mathrm{YM}}}{\delta A_{c}^{\lambda}(z)}-\delta^{\lambda-} \rho^{c}(z)\right) G_{0 c a}^{\lambda \mu}(z, x)\right\} G_{0 \mu \nu}^{-1 a b}(x, y)\right. \\
\times\left\{a_{b}^{\nu}(y)-i \int d^{4} z^{\prime} G_{0 b c^{\prime}}^{\nu \lambda^{\prime}}\left(y, z^{\prime}\right)\left(\frac{\delta S_{\mathrm{YM}}}{\delta A_{c^{\prime}}^{\lambda^{\prime}}\left(z^{\prime}\right)}-\delta^{\lambda^{\prime}-} \rho^{c^{\prime}}\left(z^{\prime}\right)\right)\right\} \\
\left.\quad-\frac{1}{2} \int d^{4} x d^{4} y\left(\frac{\delta S_{\mathrm{YM}}}{\delta A_{a}^{\mu}(x)}-\delta^{\mu-} \rho^{a}(x)\right) G_{0 a b}^{\mu \nu}(x, y)\left(\frac{\delta S_{\mathrm{YM}}}{\delta A_{b}^{\nu}(y)}-\delta^{\nu-} \rho^{b}(y)\right)\right] \\
=\operatorname{det}\left[G_{0 \mu \nu}^{-1 a b}(x, y)\right]^{-\frac{1}{2}} \exp \left[-\frac{1}{2} \int d^{4} x d^{4} y\left(\frac{\delta S_{\mathrm{YM}}}{\delta A_{a}^{\mu}(x)}-\delta^{\mu-} \rho^{a}(x)\right) G_{0 a b}^{\mu \nu}(x, y)\left(\frac{\delta S_{\mathrm{YM}}}{\delta A_{b}^{\nu}(y)}-\delta^{\nu-} \rho^{b}(y)\right)\right]
\end{gathered}
$$

where the propagator inverse for the semi-fast gluon is written as

$$
i G_{0 \mu \nu}^{-1 a b}(x, y)=\frac{\delta^{2} S_{\mathrm{YM}}}{\delta A_{a}^{\mu}(x) \delta A_{b}^{\nu}(y)}=\left\{\left[D_{\lambda} D^{\lambda}\right]^{a b} g_{\mu \nu}-\left[D_{\mu} D_{\nu}\right]^{a b}+2 g f^{a c b} F_{\mu \nu}^{c}\right\} \delta^{(4)}(x-y)
$$

with

$$
D^{-}=\partial^{-}-i g \delta A^{-}, \quad D^{i}=\partial^{i}-i g \mathcal{A}^{i}, \quad F_{+i}=-D_{i} \delta A^{-}, \quad F_{-i}=\partial^{+} \mathcal{A}_{i}, \quad F_{i j}=0
$$

in the light-cone gauge. We note that all quantities listed in (24) are matrices in the color adjoint basis.

In the rest of this step let us evaluate the determinant in the above expression (22), namely, the determinant of the propagator inverse which is expressed in the $\left(a^{-}, a^{i}\right)$ basis explicitly as

$$
i G_{0 \mu \nu}^{-1 a b}(x, y)=\left[\begin{array}{cc}
-\partial^{+2} \delta^{a b} & -\partial^{+} D_{j}^{a b}+2 g f^{a c b}\left(\partial^{+} \mathcal{A}_{j}^{c}\right) \\
-D_{i}^{a b} \partial^{+}-2 g f^{a c b}\left(\partial^{+} \mathcal{A}_{i}^{c}\right) & -\left[D_{\lambda} D^{\lambda}\right]^{a b} \delta_{i j}-\left[D_{i} D_{j}\right]^{a b}
\end{array}\right]
$$

from which one can immediately write the determinant of matrix blocks as

$$
\begin{aligned}
& \operatorname{det}\left[G_{0 \mu \nu}^{-1 a b}(x, y)\right]^{-\frac{1}{2}}=\operatorname{det}\left[-\partial^{+2} \delta^{a b}\right]^{-\frac{1}{2}} \\
& \quad \times \operatorname{det}\left[-\left[D_{\lambda} D^{\lambda}\right]^{a b} \delta_{i j}-\left[D_{i} D_{j}\right]^{a b}-\left\{-D_{i}^{a d} \partial^{+}-2 g f^{a c d}\left(\partial^{+} \mathcal{A}_{i}^{c}\right)\right\}\left(-\partial^{+2}\right)^{-1}\left\{-\partial^{+} D_{j}^{d b}+2 g f^{d c^{\prime} b}\left(\partial^{+} \mathcal{A}_{j}^{c^{\prime}}\right)\right\}\right]^{-\frac{1}{2}} .
\end{aligned}
$$

Here the first part, $\operatorname{det}\left[-\partial^{+2} \delta^{a b}\right]^{-\frac{1}{2}}$, is just an irrelevant number, and after some algebra the second part simplifies as

$$
\begin{gathered}
\operatorname{det}\left[-\left[D_{\lambda} D^{\lambda}\right]^{a b} \delta_{i j}-2 g f^{a c b}\left\{\frac{\partial_{i}}{\partial^{+}}\left(\partial^{+} \mathcal{A}_{j}^{c}\right)-\left(\partial^{+} \mathcal{A}_{i}^{c}\right) \frac{\partial_{j}}{\partial^{+}}\right\}-2 g^{2} f^{a c d} f^{d c^{\prime} b}\left\{\mathcal{A}_{i}^{c} \frac{1}{\partial^{+}}\left(\partial^{+} \mathcal{A}_{j}^{c^{\prime}}\right)-\left(\partial^{+} \mathcal{A}_{i}^{c}\right) \frac{1}{\partial^{+}} \mathcal{A}_{j}^{c^{\prime}}\right\}\right. \\
\left.-4 g^{2} f^{a c d} f^{d c^{\prime} b}\left(\partial^{+} \mathcal{A}_{i}^{c}\right) \frac{1}{\partial^{+2}}\left(\partial^{+} \mathcal{A}_{j}^{c^{\prime}}\right)\right]^{-\frac{1}{2}} \cdot
\end{gathered}
$$

Although this complicated expression looks impracticable to proceed further, we can drop many terms under the leading-log approximation as we will explain now. Let us focus on the second term $\sim\left(\partial_{i} / \partial^{+}\right)\left(\partial^{+} \mathcal{A}_{j}\right)($ the first term 
in the first curly brackets) for the moment to take an example. If one remembers that the determinant originates from the terms sandwiched by the semi-fast gluons $a_{a}^{\mu}$, one readily understands that the derivative $\partial_{i} / \partial^{+}$picks up the momenta associated with the semi-fast gluons, which is proportional to $1 / p^{+}$with $b \Lambda<\left|p^{+}\right|<\Lambda$. The terms suppressed by $1 / p^{+}$can be neglected because no logarithmic enhancement $\sim \ln [1 / b]$ arises from those terms. Because the first term $-\left[D_{\lambda} D^{\lambda}\right]^{a b} \delta_{i j}$ contains a term proportional to $p^{+}$, the logarithmic enhancing terms could appear from the part with no such $1 / p^{+}$suppression if the determinant is expanded in the power of $1 / p^{+}$. Let us next turn to the second curly brackets in (27) which contains $\mathcal{A}_{i}\left(1 / \partial^{+}\right)\left(\partial^{+} \mathcal{A}_{j}\right) \sim \mathcal{A}_{i} \mathcal{A}_{j}$ apart from the suppressed term $\mathcal{A}_{i}\left(\partial^{+} \mathcal{A}_{j}\right)\left(1 / \partial^{+}\right)$that is to be dropped by the reason we now explained. This would potentially produce logarithmic enhancing terms if expanded. Interestingly enough, however, there is a complete cancellation by the contribution from the last term in (27), i.e. $\left(\partial^{+} \mathcal{A}_{i}\right)\left(1 / \partial^{+2}\right)\left(\partial^{+} \mathcal{A}_{j}\right) \sim \mathcal{A}_{i} \mathcal{A}_{j}$ apart from the suppressed terms. Consequently we only have to retain the first term in (27) and thus arrive at

$$
\operatorname{det}\left[-\left[D_{\lambda} D^{\lambda}\right]^{a b} \delta_{i j}\right]^{-\frac{1}{2}}
$$

within the leading-log approximation. Now we make a further approximation on this. By the argument given in 26] the interaction region in which both $\bar{\rho}_{\Lambda}(\vec{x})$ and $\delta A_{a}^{-}(x)$ are nonvanishing is presumably small in the two-dimensional space spanned by $x^{+}$and $x^{-}$, that means both $p^{+}$and $p^{-}$are substantially larger than the transverse momentum scale. Then we can ignore $\partial_{i} \partial_{i}$ as compared with $2 \partial^{+} \partial^{-}$inside the determinant. Using $\partial^{+} \delta A^{-} \simeq 0$ which is valid in the light-cone gauge in the leading-log approximation $\left(\delta A^{-}\right.$has $\left|p^{+}\right|<b \Lambda$ and so $\partial^{+} \delta A^{-}$leads to contributions $\propto b$ which are neglected) the determinant is thus

$$
\operatorname{det}\left[G_{0 \mu \nu}^{-1 a b}(x, y)\right]^{-\frac{1}{2}} \simeq \operatorname{det}\left[2 \widetilde{D}^{+} \widetilde{D}^{-}\right]^{-1}=\operatorname{det}\left[\partial^{+}-i g \alpha\right]^{-1} \operatorname{det}\left[\partial^{-}-i g \delta A^{-}\right]^{-1} .
$$

Here we once moved to the covariant gauge to avoid the complexity associated with entanglement between $\partial^{i}$ which is neglected and $\mathcal{A}^{i}$ in the light-cone gauge. It should be mentioned here that the determinant of the covariant derivatives as in (29) is gauge independent as it is individually. Thus we could rotate $\delta \widetilde{A}^{-}$in the covariant gauge back to $\delta A^{-}$in the light-cone gauge without touching anything on $\operatorname{det}\left[\partial^{+}-i g \alpha\right]^{-1}$, namely, $\operatorname{det}\left[\partial^{+}-i g \delta A^{-}\right]=\operatorname{det}\left[\partial^{+}-i g \delta \widetilde{A}^{-}\right]$. In this paper we do not discuss but just discard $\operatorname{det}\left[\partial^{+}-i g \alpha\right]$ which would not play any role in the JIMWLK problem but might be important if we consider the evolution of not only the target but also the projectile.

The fact that $\operatorname{det}\left[\partial^{-}-i g \delta A^{-}\right]$is gauge independent implies that this be reduced to an irrelevant number if $\delta A^{-}$is eliminated by an appropriate gauge rotation. In other words, the determinant is expanded as a sum of all the Wilson loops which turn out to be irrelevant numbers because they sit only on the one-dimensional $x^{+}$space. If we require the periodic boundary condition in $x^{+}$, which could be suggested from the physical situation of elastic scattering, we can consider the non-trivial Wilson loop wrapping over $x^{+}$whose starting and ending points are connected by periodicity. Therefore we can expect that under the periodic boundary condition $\operatorname{det}\left[\partial^{-}-i g \delta A^{-}\right]$is given as a function of $W\left[\delta A^{-}\right]$ defined in (4).

The explicit evaluation of (29) is possible in the same way as discussed in [19], leading to the the classical color charge density as

$$
\operatorname{det}\left[\partial^{-}-i g \delta A^{-}\right]^{-1}=\int \mathcal{D} \delta \bar{\rho}_{\Lambda} w_{\Lambda}\left[\delta \bar{\rho}_{\Lambda}\right] \exp \left[-\frac{1}{g N_{c}} \int d^{3} x \operatorname{tr}\left\{\delta \bar{\rho}_{\Lambda}(\vec{x}) \ln W\left[\delta A^{-}\right](\vec{x})\right\}\right] .
$$

This is a gauge invariant expression, as we have already noted. Here the correction to the classical color charge density $\delta \bar{\rho}_{\Lambda}(\vec{x})$ distributes in $1 / \Lambda<\left|x^{-}\right|<1 / b \Lambda$ because the determinant is taken in the space spanned by the semi-fast gluon which has $b \Lambda<\left|p^{+}\right|<\Lambda$ by definition. Usually only the positive $x^{-}$distribution is chosen in accord to the retarded boundary condition which is considered as physically relevant. The combinatorial weight function associated with the spatial distribution is denoted by $w_{\Lambda}\left[\delta \bar{\rho}_{\Lambda}\right]$. This part, however, has no logarithmic enhancement $\sim \ln [1 / b]$ and is to be discarded in the leading-log approximation. It should be mentioned here that the term containing $\ln W\left[\delta A^{-}\right]$ turns out to be a shift operator acting on logarithmically enhanced terms and thus we should keep it.

(Step-3) Now that we have accomplished the Gaussian integration, we can identify the counterpart corresponding to $\mathcal{N}_{b \Lambda}[\rho]$ as a function of $\mathcal{N}_{\Lambda}[\rho]$, which leads to the renormalization group equation for $\mathcal{N}_{\Lambda}[\rho]$;

$$
\begin{aligned}
\int \mathcal{D} \rho \mathcal{N}_{b \Lambda}[\rho] e^{-i \int d^{4} x \rho^{a}(x) \delta A_{a}^{-}(x)}=\int \mathcal{D} \rho \mathcal{N}_{\Lambda}[\rho] \exp \left[\Delta S\left[\mathcal{A}\left[\bar{\rho}_{b \Lambda}\right]+\delta A^{-}, \rho\right]\right] \\
\times \int \mathcal{D} \delta \bar{\rho}_{\Lambda} \exp \left[-\frac{1}{g N_{c}} \int d^{3} x \operatorname{tr}\left\{\delta \bar{\rho}_{\Lambda}(\vec{x}) \ln W[i \delta / \delta \rho](\vec{x})\right\}\right] e^{-i \int d^{4} x \rho^{a}(x) \delta A_{a}^{-}(x)},
\end{aligned}
$$


where $\Delta S$ is the rest of the Gaussian integration (22) given by

$$
\Delta S\left[\mathcal{A}\left[\bar{\rho}_{b \Lambda}\right]+\delta A^{-}, \rho\right]=-\frac{1}{2}\left(\frac{\delta S_{\mathrm{YM}}}{\delta A_{a}^{\mu}(x)}-\delta^{\mu-} \rho^{a}(x)\right) G_{0 a b}^{\mu \nu}(x, y)\left(\frac{\delta S_{\mathrm{YM}}}{\delta A_{b}^{\nu}(y)}-\delta^{\nu-} \rho^{b}(y)\right) .
$$

(Step-4) Then the evolution equation for the weight functional $\mathcal{W}_{\Lambda}\left[\bar{\rho}_{\Lambda}\right]$ follows immediately from (31) as

$$
\begin{aligned}
\int \mathcal{D} \rho e^{-i \int d^{4} x \rho^{a}(x) \delta A_{a}^{-}(x)} \int \mathcal{D} \bar{\rho}_{b \Lambda}\left[\mathcal{W}_{b \Lambda}\left[\bar{\rho}_{b \Lambda}\right] e^{-\frac{1}{g N_{c}} \int d^{3} x \operatorname{tr}\left\{\bar{\rho}_{b \Lambda}(\vec{x}) \ln W[-i \delta / \delta \rho](\vec{x})\right\}}\right. \\
\left.\quad-\mathcal{W}_{\Lambda}\left[\bar{\rho}_{b \Lambda}\right] e^{-\frac{1}{g N_{c}} \int d^{3} x \operatorname{tr}\left\{\delta \bar{\rho}_{\Lambda}(\vec{x}) \ln W[-i \delta / \delta \rho](\vec{x})\right\}} e^{\Delta S\left[\mathcal{A}\left[\bar{\rho}_{b \Lambda}\right]+\delta A^{-}, \rho\right]} e^{-\frac{1}{g N_{c}} \int d^{3} x \operatorname{tr}\left\{\bar{\rho}_{\Lambda}(\vec{x}) \ln W[-i \delta / \delta \rho](\vec{x})\right\}}\right] \delta[\rho]=0 .
\end{aligned}
$$

Here we defined the integration $\int \mathcal{D} \bar{\rho}_{b \Lambda}=\int \mathcal{D} \bar{\rho}_{\Lambda} \int \mathcal{D} \delta \bar{\rho}_{\Lambda}$, and the weight functional is extended as [20]

$$
\mathcal{W}_{\Lambda}\left[\bar{\rho}_{b \Lambda}\right]=\mathcal{W}_{\Lambda}\left[\bar{\rho}_{\Lambda}\right] \prod_{1 / \Lambda<x^{-}<1 / b \Lambda} \delta\left[\delta \bar{\rho}_{\Lambda}\right]
$$

We leave $\delta A^{-}$in $\Delta S$ as it is (not replaced by $-i \delta / \delta \rho$ ) on purpose to avoid confusion in the approximation we will make later.

In the dense regime where $\bar{\rho}_{\Lambda}$ takes a large value, we can make an important approximation here, i.e. we can expand the Wilson line term as

$$
\begin{aligned}
& -\frac{1}{g N_{c}} \int d^{3} x \operatorname{tr}\left\{\bar{\rho}_{\Lambda}(\vec{x}) \ln W[-i \delta / \delta \rho](\vec{x})\right\} \\
\simeq & -\int d^{4} x \bar{\rho}_{\Lambda}^{a}(\vec{x}) \frac{\delta}{\delta \rho^{a}(x)}-\frac{g}{2 N_{c}} \int d^{3} x(\mathcal{P}-1) \int d x^{+} d y^{+} \operatorname{tr}\left\{\bar{\rho}_{\Lambda}(\vec{x}) \frac{\delta}{\delta \rho\left(x^{+}\right)} \frac{\delta}{\delta \rho\left(y^{+}\right)}\right\}+\cdots,
\end{aligned}
$$

where the higher-order contributions involve more derivatives $\delta / \delta \rho$, so that they are suppressed when $\bar{\rho}_{\Lambda}$ or $\rho$ is large. [ $\rho$ is of the same order as $\bar{\rho}_{\Lambda}$ as we will see shortly.] Here $\mathcal{P}$ stands for time ordering as in (4). The first term in (35) placed on the exponential leads to the shift operator which makes $f[\rho]$ be $f\left[\rho-\bar{\rho}_{\Lambda}\right]$. The second term is vanishing under the second-order perturbation that the terms up to the quadratic order in $\delta A^{-}$or $\delta / \delta \rho^{a}$ are kept; the derivatives in the second term should act on the function of $\rho^{a}(x)$ arising as a result of the operation by the first term. Then the delta functional shifted by $\bar{\rho}_{\Lambda}^{a}(\vec{x})$ leads to $\rho^{a}(x)=\bar{\rho}_{\Lambda}^{a}(\vec{x})$ which is $x^{+}$independent. Once $\rho^{a}(x)$ loses the $x^{+}$dependence, then only the first term in the expansion (35) remains, which signifies that non-commutativity becomes completely irrelevant. Therefore we insist that the expansion (35) is the key equation to understand how the non-commutativity is disregarded in the JIMWLK problem in the high density regime. In App. \$ we will comment on the connection of (35) to an approximation made in the conventional derivation of the JIMWLK equation.

In evaluating (33), keeping only the first term in the expansions like (35), one can simplify it as

$$
\int \mathcal{D} \rho e^{-i \int d^{4} x \rho^{a}(x) \delta A_{a}^{-}(x)} \int \mathcal{D} \bar{\rho}_{b \Lambda}\left[\mathcal{W}_{b \Lambda}\left[\bar{\rho}_{b \Lambda}\right]-\mathcal{W}_{\Lambda}\left[\bar{\rho}_{b \Lambda}\right] e^{\Delta S\left[\mathcal{A}\left[\bar{\rho}_{b \Lambda}\right]+\delta A^{-}, \rho+\delta \bar{\rho}_{\Lambda}\right]}\right] \delta\left[\rho-\bar{\rho}_{\Lambda}-\delta \bar{\rho}_{\Lambda}\right]=0 .
$$

One can readily complete the $\rho$-integration here, then replace $\delta A^{-}$in $\Delta S$ by the derivative in $\bar{\rho}_{b \Lambda}=\bar{\rho}_{\Lambda}+\delta \bar{\rho}_{\Lambda}$, and move the derivative onto $\mathcal{W}_{\Lambda}\left[\bar{\rho}_{b \Lambda}\right]$ by the integration by parts. Finally we get the evolution equation in the form of

$$
\mathcal{W}_{b \Lambda}\left[\bar{\rho}_{b \Lambda}\right]-e^{\Delta S\left[\mathcal{A}\left[\bar{\rho}_{b \Lambda}\right]-i \delta / \delta \bar{\rho}_{b \Lambda}, \bar{\rho}_{\Lambda}\right]} \mathcal{W}_{\Lambda}\left[\bar{\rho}_{b \Lambda}\right]=0 .
$$

It should be noted that the $\bar{\rho}_{b \Lambda}$-derivative hits all $\bar{\rho}_{b \Lambda}$ from the far left, that is, it acts on $\bar{\rho}_{\Lambda}$ in $\Delta S$ as well as on $\bar{\rho}_{b \Lambda}$ in $\mathcal{W}_{\Lambda}\left[\bar{\rho}_{b \Lambda}\right]$. A subtle point is that $\Delta S$ contains not only $\bar{\rho}_{b \Lambda}$ but also $\bar{\rho}_{\Lambda}$. This is because the origin of $\bar{\rho}_{\Lambda}$ in $\Delta S$ is traced back to the eikonal coupling $\sim \rho^{a} a_{a}^{-}$for semi-fast gluons. The integration over $a_{a}^{\mu}$ leads to the renormalization $\delta \bar{\rho}_{\Lambda}$ for soft gluons, but not for semi-fast gluons themselves. It might seem then that taking the limit of $b \rightarrow 1$ in $\Delta S$ is a subtle business. This does not matter in fact, however, because there remains no contribution from the $\bar{\rho}_{b \Lambda}$-derivative hitting $\bar{\rho}_{b \Lambda}$ inside $\Delta S$ as we shall confirm explicitly below. [We will see another reasoning for that in App. @]

The rest of this section would be devoted to illustrating the evaluation of $\Delta S\left[\mathcal{A}\left[\bar{\rho}_{b \Lambda}\right]-i \delta / \delta \bar{\rho}_{b \Lambda}, \bar{\rho}_{\Lambda}\right]$. At this point we realize that $\Delta S\left[\mathcal{A}\left[\bar{\rho}_{b \Lambda}\right]-i \delta / \delta \bar{\rho}_{b \Lambda}, \bar{\rho}_{\Lambda}\right]$ is the same quantity calculated in Sec. 6 of [26], while (32) was different. We can borrow the results from [26] but shall try to make our calculations in a self-contained fashion. We will work in the light-cone gauge, which eventually leads to the same formula as the calculation in the covariant gauge in [26], of course. By using $\partial^{+} \delta A^{-}=0$ one can easily see

$$
\left.\frac{\delta S_{\mathrm{YM}}}{\delta A_{a}^{\mu}}\right|_{\mathcal{A}\left[\bar{\rho}_{b \Lambda}\right]-i \delta / \delta \bar{\rho}_{b \Lambda}}-\delta^{\mu-} \bar{\rho}_{\Lambda}=\delta^{\mu i} 2 i g \partial^{+}\left\{f^{a b c} \frac{\delta}{\delta \bar{\rho}_{b \Lambda}^{b}} \mathcal{A}_{c}^{i}\left[\bar{\rho}_{b \Lambda}\right]\right\}+\delta^{\mu-} \delta \bar{\rho}_{\Lambda}
$$


in the light-cone gauge. One can also express it in terms of the quantities in the covariant gauge by means of

$$
f^{a b c} \frac{\delta}{\delta \bar{\rho}_{b \Lambda}^{b}} \mathcal{A}_{c}^{i}=\frac{2}{i N_{c}} \operatorname{tr}\left[T^{a} \frac{\delta}{\delta \bar{\rho}_{b \Lambda}} \mathcal{A}^{i}\right]=\frac{1}{g}\left(\partial^{i} \frac{\delta}{\delta \bar{\rho}_{b \Lambda}^{b}}\right)\left(V_{x^{-},-\infty}^{\dagger}\right)_{b a} .
$$

Here we substituted (11) and rotated the $\bar{\rho}_{b \Lambda^{-}}$-derivative by $V_{x^{-},-\infty}$ to make it in the covariant gauge and used a formula $V_{a b}=\left(1 / N_{c}\right) \operatorname{tr}\left[T^{a} V T^{b} V^{\dagger}\right]$. Also we performed the integration by parts to move $\partial^{i}$ onto $\delta / \delta \bar{\rho}_{b \Lambda}$. Although we skip the detailed explanations, we can use the free propagator, i.e. the propagator in the absence of the background field $\mathcal{A}_{a}^{i}(\vec{x})$, if we employ the light-cone gauge. This is, roughly speaking, because in the light-cone gauge $\mathcal{A}_{a}^{i}(\vec{x})$ has no singularity but only a discontinuity at $x^{-}=0$ which does not affect the propagator [18]. The free propagator can be further approximated in the interested region as 26$]$

$$
G_{0 a b}^{i j}(x, y) \simeq \delta^{i j} \delta^{a b} \frac{d \tau}{4 \pi} \delta^{(2)}\left(\boldsymbol{x}_{\perp}-\boldsymbol{y}_{\perp}\right),
$$

where $d \tau=\ln [1 / b]$. The other components, $G_{0 a b}^{i-}, G_{0 a b}^{-i}$, and $G_{0 a b}^{--}$, are suppressed by $1 / p^{+}$as compared with $G_{0 a b}^{i j}$ and we drop them in the leading-log approximation. Therefore, under this approximation, we can neglect $\delta^{\mu-} \delta \bar{\rho}_{\Lambda}$ in (38) because no logarithmic enhancement results from the terms with $\delta^{\mu-} \delta \bar{\rho}_{\Lambda}$ in $\Delta S$.

Because the first term in (38) is a total derivative in $x^{-}$and the approximate propagator (40) does not depend on $x^{-}$, the integrations over $x^{-}$and $y^{-}$are almost trivial, so that only the surface terms at $x^{-}, y^{-}= \pm \infty$ remain. For instance, the integration of the first term in (38) with respect to $x^{-}$results in

$$
\delta^{\mu i} 2 i\left[\left(\partial^{i} \frac{\delta}{\delta \bar{\rho}_{\tau}^{b}\left(x_{\tau}^{-}, \boldsymbol{x}_{\perp}\right)}\right) V_{b a}^{\dagger}\left(\boldsymbol{x}_{\perp}\right)-\left(\partial^{i} \frac{\delta}{\delta \bar{\rho}_{\tau}^{a}\left(0, \boldsymbol{x}_{\perp}\right)}\right)\right] .
$$

In the above we have referred to the separation scale by using the rapidity variable $\tau$ instead of $\Lambda$ or $b \Lambda$ and have used the fact that $\bar{\rho}_{\tau}(\vec{x})$ has a support $0 \lesssim x^{-} \lesssim x_{\tau}^{-}$. The matrix $V$ is $V_{x_{\tau}^{-},-\infty} \simeq V_{+\infty,-\infty}$ with the definition (12). The integrand does not depend on time and thus the $x^{+}$and $y^{+}$integrations are absorbed in the redefinition as $\int d x^{+} \delta / \delta \bar{\rho}_{\tau}(x) \rightarrow \delta / \delta \bar{\rho}_{\tau}(\vec{x})$ (see (6.8) in [26]). After all $\Delta S$ takes a form of

$$
\begin{aligned}
\Delta S=\frac{d \tau}{2 \pi} \int d^{2} \boldsymbol{x}_{\perp}\left[\left(\partial^{i} \frac{\delta}{\delta \bar{\rho}_{\tau}^{a}\left(x_{\tau}^{-}, \boldsymbol{x}_{\perp}\right)}\right)\left(\partial^{i} \frac{\delta}{\delta \bar{\rho}_{\tau}^{a}\left(x_{\tau}^{-}, \boldsymbol{x}_{\perp}\right)}\right)-\left(\partial^{i} \frac{\delta}{\delta \bar{\rho}_{\tau}^{a}\left(x_{\tau}^{-}, \boldsymbol{x}_{\perp}\right)}\right)\left(\partial^{i} \frac{\delta}{\delta \bar{\rho}_{\tau}^{b}\left(0, \boldsymbol{x}_{\perp}\right)}\right) V_{a b}^{\dagger}\left(\boldsymbol{x}_{\perp}\right)\right. \\
\left.-\left(\partial^{i} \frac{\delta}{\delta \bar{\rho}^{a}\left(0, \boldsymbol{x}_{\perp}\right)}\right)\left(\partial^{i} \frac{\delta}{\delta \bar{\rho}^{b}\left(x_{\tau}^{-}, \boldsymbol{x}_{\perp}\right)}\right) V_{a b}\left(\boldsymbol{x}_{\perp}\right)+\left(\partial^{i} \frac{\delta}{\delta \bar{\rho}^{a}\left(0, \boldsymbol{x}_{\perp}\right)}\right)\left(\partial^{i} \frac{\delta}{\delta \bar{\rho}^{a}\left(0, \boldsymbol{x}_{\perp}\right)}\right)\right],
\end{aligned}
$$

which is nothing but (6.5) in [26]. It is apparent from this expression that the $\bar{\rho}_{\tau}$-derivative does not act on $\bar{\rho}_{\tau}$ in $\Delta S$ because $\delta V_{a b}^{\dagger} / \delta \bar{\rho}_{\tau}^{a}\left(x_{\tau}^{-}\right) \propto\left(T^{a}\right)_{a c} V_{c b}^{\dagger}=0$ and $\delta V_{a b}^{\dagger} / \delta \bar{\rho}_{\tau}^{b}(0) \propto V_{a c}^{\dagger}\left(T^{b}\right)_{c b}=0$, etc. Then in exactly the same way as in [26], we can change the variable from $\bar{\rho}_{\tau}^{a}(\vec{x})$ to $\alpha_{\tau}^{a}(\vec{x})$ by (10) and with the help of relations,

$$
\frac{\delta}{\delta \alpha_{\tau}^{a}\left(0, \boldsymbol{x}_{\perp}\right)}=\frac{\delta}{\delta \alpha_{\tau}^{b}\left(x_{\tau}^{-}, \boldsymbol{x}_{\perp}\right)} V_{b a}^{\dagger}\left(\boldsymbol{x}_{\perp}\right)=V_{a b}\left(\boldsymbol{x}_{\perp}\right) \frac{\delta}{\delta \alpha_{\tau}^{b}\left(x_{\tau}^{-}, \boldsymbol{x}_{\perp}\right)},
$$

it is immediate to confirm that we finally reach the JIMWLK equation (13). Let us focus on the second term in (42) to take an example. Using (10), (14), and (43) in order we have

$$
\begin{aligned}
& -\frac{d \tau}{2 \pi} \int d^{2} \boldsymbol{x}_{\perp}\left(\partial^{i} \frac{\delta}{\delta \bar{\rho}_{\tau}^{a}\left(x_{\tau}^{-}, \boldsymbol{x}_{\perp}\right)}\right)\left(\partial^{i} \frac{\delta}{\delta \bar{\rho}_{\tau}^{b}\left(0, \boldsymbol{x}_{\perp}\right)}\right) V_{a b}^{\dagger}\left(\boldsymbol{x}_{\perp}\right) \\
= & -\frac{d \tau}{2 \pi} \int d^{2} \boldsymbol{x}_{\perp} d^{2} \boldsymbol{y}_{\perp} d^{2} \boldsymbol{z}_{\perp}\left\langle\boldsymbol{z}_{\perp}\left|\frac{\boldsymbol{\partial}_{\perp}}{\boldsymbol{\partial}_{\perp}^{2}}\right| \boldsymbol{x}_{\perp}\right\rangle \cdot\left\langle\boldsymbol{z}_{\perp}\left|\frac{\boldsymbol{\partial}_{\perp}}{\boldsymbol{\partial}_{\perp}^{2}}\right| \boldsymbol{y}_{\perp}\right\rangle \frac{\delta}{\delta \alpha_{\tau}^{a}\left(x_{\tau}^{-}, \boldsymbol{x}_{\perp}\right)} \frac{\delta}{\delta \alpha_{\tau}^{b}\left(0, \boldsymbol{y}_{\perp}\right)} V_{a b}^{\dagger}\left(\boldsymbol{z}_{\perp}\right) \\
= & -\frac{d \tau}{(2 \pi)^{3}} \int d^{2} \boldsymbol{x}_{\perp} d^{2} \boldsymbol{y}_{\perp} d^{2} \boldsymbol{z}_{\perp} \mathcal{K}\left(\boldsymbol{x}_{\perp}, \boldsymbol{y}_{\perp}, \boldsymbol{z}_{\perp}\right) \frac{\delta}{\delta \alpha_{\tau}^{a}\left(x_{\tau}^{-}, \boldsymbol{x}_{\perp}\right)} V_{c b}\left(\boldsymbol{y}_{\perp}\right) \frac{\delta}{\delta \alpha_{\tau}^{b}\left(x_{\tau}^{-}, \boldsymbol{y}_{\perp}\right)} V_{a c}^{\dagger}\left(\boldsymbol{z}_{\perp}\right),
\end{aligned}
$$

which is identical with the second term inside the square brackets in the evolution kernel (14).

Before closing this section, we shall comment upon the meaning of (43). We can easily validate these relations supposing that it is only $V$ or $V^{\dagger}$ on which the $\alpha_{\tau}$-derivatives act. It does not mean, however, that $\mathcal{W}_{\tau}\left[\bar{\rho}_{\tau}\right]$ is a function of $V$ and $V^{\dagger}$. In fact, the derivatives can be moved from on $\mathcal{W}_{\tau}\left[\bar{\rho}_{\tau}\right]$ to on $\mathcal{O}\left[\bar{\rho}_{\tau}\right]$ on averaging over $\alpha_{\tau}\left(\right.$ or $\left.\bar{\rho}_{\tau}\right)$ in (77). Thus, it is sufficient to require $\mathcal{O}\left[\bar{\rho}_{\tau}\right]$ written in terms of $V$ and $V^{\dagger}$, which is actually the case if we consider the scattering between the color glass condensate and the projectile consisting of partons in the eikonal approximation. 
From the practical point of view we can say that the relations of (43) would not lose generality of the evolution equation.

This treatment may well arise a question about the ordering problem. It is obvious from (44) that $\delta V_{c b}\left(\boldsymbol{y}_{\perp}\right) / \delta \alpha_{\tau}^{a}\left(\boldsymbol{x}_{\perp}\right)$ is nonvanishing and so $\delta / \delta \alpha_{\tau}^{a}\left(x_{\tau}^{-}\right)$and $\delta / \delta \alpha_{\tau}^{b}(0)$ do not commute after (43) is applied, while they should be commutative by construction. This is not a paradox, however. For example, in (44), there is certainly a non-zero contribution from $\delta V_{c b}\left(\boldsymbol{y}_{\perp}\right) / \delta \alpha_{\tau}^{a}\left(\boldsymbol{x}_{\perp}\right)$, but such a term is precisely compensated for by the term arising from non-commutativity among $\alpha_{\tau}$-derivatives acting on $V$ or $V^{\dagger}$, that is,

$$
\left[\frac{1}{i} \frac{\delta}{\delta \alpha_{\tau}^{a}\left(\boldsymbol{x}_{\perp}\right)}, \frac{1}{i} \frac{\delta}{\delta \alpha_{\tau}^{b}\left(\boldsymbol{y}_{\perp}\right)}\right]=-i g f^{a b c} \frac{1}{i} \frac{\delta}{\delta \alpha_{\tau}^{c}\left(\boldsymbol{x}_{\perp}\right)} \delta^{(2)}\left(\boldsymbol{x}_{\perp}-\boldsymbol{y}_{\perp}\right),
$$

if these derivatives are supposed to act on $V$ or $V^{\dagger}$. Therefore, there is no ordering problem at all unless the quantities in (43) are split apart; in (43) an ordering like $\left(\delta / \delta \alpha_{\tau}^{b}\left(\boldsymbol{y}_{\perp}\right)\right)\left(\delta / \delta \alpha_{\tau}^{a}\left(\boldsymbol{x}_{\perp}\right)\right) V_{c b}\left(\boldsymbol{y}_{\perp}\right)$ is not allowed, which generates extra terms and causes the ordering ambiguity. In other words, the ordering realizing in the JIMWLK equation (13) is determined uniquely in a way that (45) would bring about no ordering problem.

In the present calculation we did not treat the virtual term $\sigma^{a}$ (see (A9)) in an explicit manner. The virtual term is, in fact, included implicitly in the correct ordering as pointed out in [26] and it will be clearer in the discussions in App. A

\section{CONCLUSIONS}

We have derived the JIMWLK equation in the functional integral formalism. Our starting point is different from the conventional derivation; we used a different representation of the QCD generating functional consisting of the density of states and the simple eikonal coupling. In this way, we could clarify how the non-commutative nature of quantum color charge density becomes irrelevant in the approximation that the color charge density is large. Actually the expansion of the Wilson line in the non-local source term has turned out to be responsible for loss of non-commutativity. We further discuss in App. A some connections between the approximations made in the new derivation and their counterparts in the conventional derivation. The comparison between two different methods would give us a deeper understanding of those approximations.

The next step would be how we can improve the approximation to handle non-commutativity in the functional integral formalism. The commutation relation between the functional derivatives with respect to the classical gauge field (see (45)) originates from that the derivatives act on the gauge invariant Wilson lines, and there is no difficulty in this case. In contrast, in the case of the commutation relation between the color charge density operators, the functional integral description for that is non-trivial because the color charge density in the functional integral is not an operator like the derivative but a given classical field. From our analysis we would presume that the non-local source term written in terms of the Wilson line takes care of this, that is, if one is capable to take full account of the source term, one could reach a general evolution equation in terms of classical quantities alone. In our formalism such a manipulation would be possible, at least in principle, by diagonalizing the classical color source, and rotating it back in color space. We are making progress in this direction. The point is, once it will be accomplished, the quantization is automatic and there is no need to impose the commutation relation between color charge density like in 26.

Although it is far from obvious whether the renormalization group equation is closed in a color glass condensate formalism in an intermediate region between the dense and dilute regimes, it would be a challenging problem to attack it in the functional integral formalism. We believe that the present formulation would provide a useful information for that purpose.

\section{Acknowledgments}

The author thanks L. McLerran and Y. Hatta for discussions. He also thanks E. Iancu for a careful reading through the manuscript and useful comments. This work was supported by the RIKEN BNL Research Center and the U.S. Department of Energy (D.O.E.) under cooperative research agreement \#DE-AC02-98CH10886.

\section{APPENDIX A: COMPARISON TO THE CONVENTIONAL DERIVATION}

In this paper we have elaborated the derivation of the JIMWLK equation in the framework with the source terms consisting of the density of states and the simple eikonal coupling. Now, let us look over the conventional derivation to grasp some connections between two different strategies. 
The derivation we address here is not faithful to the original one in literatures. We shall rearrange the calculations and put our emphasis on some aspects which are related to our derivation in the text, though the main building blocks are not quite new. The weight functional $\mathcal{W}_{\Lambda}\left[\bar{\rho}_{\Lambda}\right]$ changes according to $\Lambda$ in a way to keep the generating functional $Z$ intact, from which the evolution equation follows. That is, $Z$ can be written at arbitrary separation scale $b \Lambda$ as in (11). We shall take (Step-1), (Step-2), and (Step-3) respectively as in the derivation we addressed previously.

(Step-1) The gauge fields are decomposed into the semi-fast gluon $a_{a}^{\mu}$ with $b \Lambda<\left|p^{+}\right|<\Lambda$, the classical solution $\mathcal{A}_{a}^{i}\left[\bar{\rho}_{\Lambda}+\delta \rho\right]$, and the rest $\delta A_{a}^{\mu}$ with $\left|p^{+}\right|<b \Lambda$, and then the action, $S=S_{\mathrm{YM}}+S_{W}$, is expanded up to the quadratic order in terms of $a_{a}^{\mu}$;

$$
\begin{aligned}
\int^{\Lambda} \mathcal{D} A \delta\left[A^{+}\right] \exp \left[i S\left[A, \bar{\rho}_{\Lambda}\right]\right] & \simeq \int^{b \Lambda} \mathcal{D} \delta A \delta\left[\delta A^{+}\right] \int_{b \Lambda}^{\Lambda} \mathcal{D} a \delta\left[a^{+}\right] \exp \left[i S\left[\mathcal{A}+\delta A, \bar{\rho}_{\Lambda}\right]\right] \\
& \times \exp \left[i \int d^{4} x \frac{\delta S}{\delta A_{a}^{\mu}(x)} a_{a}^{\mu}(x)+\frac{i}{2} \int d^{4} x d^{4} y \frac{\delta^{2} S}{\delta A_{a}^{\mu}(x) \delta A_{b}^{\nu}(y)} a_{a}^{\mu}(x) a_{b}^{\nu}(y)\right] .
\end{aligned}
$$

It should be mentioned that in this case the action contains $S_{W}\left[A^{-}, \bar{\rho}_{\Lambda}\right]$ given by (5) that generates non-local terms in $x^{+}$. This is an important difference from what we have seen in our derivation in Sec. III]

In the dense regime where $\delta A_{a}^{\mu}$ is small (A1) can be further expanded in terms of $\delta A_{a}^{\mu}$ to read the quantum corrections to the source as

$$
\int_{b \Lambda}^{\Lambda} \mathcal{D} a \delta\left[a^{+}\right] \exp \left[-\frac{1}{2} \int d^{4} x d^{4} y a_{a}^{\mu}(x) G_{\mu \nu}^{-1 a b}(x, y) a_{b}^{\nu}(y)-i \int d^{4} x \delta \rho^{a}(x)\left(-a_{a}^{-}(x)+\delta A_{a}^{-}(x)\right)\right],
$$

where we have used the equations of motion including the source terms, i.e.

$$
\left.\frac{\delta S\left[A, \bar{\rho}_{\Lambda}\right]}{\delta A_{a}^{\mu}(x)}\right|_{\mathcal{A}\left[\bar{\rho}_{\Lambda}+\delta \rho\right]}=\delta \rho^{a}(x)
$$

and we have defined the corrections to the source,

$$
\delta \rho^{a}(x)=\delta \rho^{(1) a}(x)+\delta \rho^{(2) a}(x)=-\left.\int d^{4} y \frac{\delta^{2} S}{\delta A_{a}^{-}(x) \delta A_{b}^{\nu}(y)}\right|_{\mathcal{A}} a_{b}^{\nu}(y)-\left.\frac{1}{2} \int d^{4} y d^{4} z \frac{\delta^{3} S}{\delta A_{a}^{-}(x) \delta A_{b}^{\nu}(y) \delta A_{c}^{\lambda}(z)}\right|_{\mathcal{A}} a_{b}^{\nu}(y) a_{c}^{\lambda}(z) .
$$

This approximation that one keeps only the linear term, $-i \delta \rho^{a}(x) \delta A_{a}^{-}(x)$, corresponds to the expansion of (35) and is responsible for loss of non-commutativity. In writing (A2) we have defined the propagator for $a_{a}^{\mu}$ with $S_{W}$ included (that makes a difference from $G_{0 \mu \nu}^{-1 a b}$ appearing in Sec. IIIB) as

$$
i G_{\mu \nu}^{-1 a b}\left[\bar{\rho}_{\Lambda}\right](x, y)=\left.\frac{\delta^{2} S}{\delta A_{a}^{\mu}(x) \delta A_{b}^{\nu}(y)}\right|_{\mathcal{A}},
$$

where the argument $\bar{\rho}_{\Lambda}$ of $G_{\mu \nu}^{-1 a b}$ refers to the source contained in $S_{W}$.

(Step-2) The generating functional with the separation scale $\Lambda$ is now rewritten in a form from which we can identify the weight functional at scale $b \Lambda$, that is,

$$
\begin{aligned}
& \int \mathcal{D} \bar{\rho}_{\Lambda} \mathcal{W}_{\Lambda}\left[\bar{\rho}_{\Lambda}\right] \int^{\Lambda} \mathcal{D} \delta A \delta\left[\delta A^{+}\right] e^{i S\left[\mathcal{A}+\delta A, \bar{\rho}_{\Lambda}\right]} \\
= & \int \mathcal{D} \bar{\rho}_{\Lambda} \mathcal{W}_{\Lambda}\left[\bar{\rho}_{\Lambda}\right] \int_{b \Lambda}^{\Lambda} \mathcal{D} a \delta\left[a^{+}\right] e^{-\frac{1}{2} \int d^{4} x d^{4} y a_{a}^{\mu}(x) G_{\mu \nu}^{-1 a b}(x, y) a_{b}^{\nu}(y)+i \int d^{4} x \delta \rho^{a}(x) a_{a}^{-}(x)} \int^{b \Lambda} \mathcal{D} \delta A \delta\left[\delta A^{+}\right] e^{i S\left[\mathcal{A}+\delta A, \bar{\rho}_{\Lambda}+\delta \rho\right]},
\end{aligned}
$$

where the last term in (A2) gives the renormalization of the source $\bar{\rho}_{\Lambda} \rightarrow \bar{\rho}_{\Lambda}+\delta \rho$ in $S$ in the above expression. We shall introduce an auxiliary variable $\bar{\rho}_{b \Lambda}$ which is constrained as $\bar{\rho}_{b \Lambda}=\bar{\rho}_{\Lambda}+\delta \rho$. It should be worth mentioning that $\delta \rho$ is a function of $a_{a}^{\mu}$ and should be interpreted as the quantum color charge density in a sense in discussions in Sec. IIIA In the dense approximation, however, as we have explained before, $\delta A^{-}$is taken only up to the first order and at that stage we have already lost the discrimination between quantum and classical color charge density. By introducing $\bar{\rho}_{b \Lambda}$ and integrating over $\bar{\rho}_{\Lambda}$, we reach

$$
\begin{aligned}
& \int \mathcal{D} \bar{\rho}_{b \Lambda} \int \mathcal{D} \bar{\rho}_{\Lambda} \mathcal{W}_{\Lambda}\left[\bar{\rho}_{\Lambda}\right] \int_{b \Lambda}^{\Lambda} \mathcal{D} a \delta\left[a^{+}\right] e^{-\frac{1}{2} \int d^{4} x d^{4} y a_{a}^{\mu}(x) G_{\mu \nu}^{-1 a b}(x, y) a_{b}^{\nu}(y)+i \int d^{4} x \delta \rho^{a}(x) a_{a}^{-}(x)} \delta\left[\bar{\rho}_{b \Lambda}-\bar{\rho}_{\Lambda}-\delta \rho\right] \\
= & \int \mathcal{D} \bar{\rho}_{b \Lambda} \int_{b \Lambda}^{\Lambda} \mathcal{D} a \delta\left[a^{+}\right] e^{-\frac{1}{2} \int d^{4} x d^{4} y a_{a}^{\mu}(x) G_{\mu \nu}^{-1 a b}(x, y) a_{b}^{\nu}(y)+i \int d^{4} x \delta \rho^{a}(x) a_{a}^{-}(x)} \mathcal{W}_{\Lambda}\left[\bar{\rho}_{b \Lambda}-\delta \rho\right],
\end{aligned}
$$


where $\bar{\rho}_{\Lambda}$ inside $G_{\mu \nu}^{-1 a b}(x, y)$ must be altered according to $\bar{\rho}_{\Lambda} \rightarrow \bar{\rho}_{b \Lambda}-\delta \rho$, though this could be ignored in the Gaussian approximation with respect to $a_{a}^{\mu}(x)$. Otherwise, the already quadratic term $-\frac{1}{2} a_{a}^{\mu} G_{\mu \nu}^{-1 a b} a_{b}^{\nu}$ could produce higher order terms.

Here we shall remark that the role of the $\delta \rho^{a}(x) a_{a}^{-}(x)$ term should deserve further investigations. If we treat it in the Gaussian approximation, it cancels $G_{-\mu}^{-1 a b}$ and $G_{\mu-}^{-1 a b}$, and is irrelevant to the final results. It has been anticipated in [23], however, that the complete integration over $a_{a}^{\mu}(x)$ including the full Yang-Mills action would result in the Wess-Zumino term for $\delta \rho^{a}(x)$. In this paper, we shall just drop $\delta \rho^{a}(x) a_{a}^{-}(x)$ hereafter. This treatment is valid in deriving the JIMWLK equation in the dense regime.

Expanding the above expression in terms of $\delta \rho$ (with the $\delta \rho^{a}(x) a_{a}^{-}(x)$ term dropped), we have

$$
\mathcal{W}_{b \Lambda}\left[\bar{\rho}_{b \Lambda}\right]=\mathcal{W}_{\Lambda}\left[\bar{\rho}_{b \Lambda}\right]-\int d^{4} x \frac{\delta}{\delta \bar{\rho}_{b \Lambda}^{a}(x)}\left(\mathcal{W}_{\Lambda}\left[\bar{\rho}_{b \Lambda}\right] \sigma^{a}(x)\right)+\frac{1}{2} \int d^{4} x d^{4} y \frac{\delta^{2}}{\delta \bar{\rho}_{b \Lambda}^{a}(x) \delta \bar{\rho}_{b \Lambda}^{b}(y)}\left(\mathcal{W}_{\Lambda}\left[\bar{\rho}_{b \Lambda}\right] \chi^{a b}(x, y)\right)
$$

where the correlation functions are defined as

$$
\begin{gathered}
\sigma^{a}(x)=\left\langle\delta \rho^{(2) a}(x)\right\rangle=-\frac{1}{2} \int d^{4} y d^{4} z \frac{\delta^{3} S}{\delta A_{a}^{-}(x) \delta A_{b}^{\nu}(y) \delta A_{c}^{\lambda}(z)} \cdot G_{b c}^{\nu \lambda}(y, z) \\
\chi^{a b}(x, y)=\left\langle\delta \rho^{(1) a}(x) \delta \rho^{(1) b}(y)\right\rangle=\int d^{4} y d^{4} z d^{4} w \frac{\delta^{2} S}{\delta A_{a}^{-}(x) \delta A_{c}^{\lambda}(z)} \cdot \frac{\delta^{2} S}{\delta A_{b}^{-}(y) \delta A_{d}^{\sigma}(w)} \cdot G_{c d}^{\lambda \sigma}(z, w)
\end{gathered}
$$

within the Gaussian approximation. This is exactly the same expression as (3.53) in [16] and the evaluation of these correlation functions goes just in the same manner. We shall comment that in this case the determinant as a result of the Gaussian integration would not lead to the determinant (30) because the propagator defined in this section does not contain $\delta A^{-}$in it.

As we have already mentioned, we could have ignored the difference by $\delta \rho$ inside the propagator within the Gaussian approximation. Then the $\bar{\rho}_{b \Lambda}$-derivatives in (A8) would not hit the $\bar{\rho}_{\Lambda}$ dependence in the propagator. This fact seems to correspond to the arguments in Sec. IIIB that the $\bar{\rho}_{b \Lambda}$-derivatives vanish acting on $\bar{\rho}_{b \Lambda}$ in $\Delta S$. We cannot see the mathematical correspondence transparently, however, for the propagator A5 involves non-local contributions from $S_{W}\left[A^{-}, \bar{\rho}\right]$.

It is known that the explicit evaluation reveals an interesting relation,

$$
\frac{1}{2} \int d^{4} y \frac{\delta \chi^{a b}(x, y)}{\delta \bar{\rho}_{b \Lambda}^{b}(y)}=\sigma^{a}(x)
$$

from which (A8) simplifies as

$$
\frac{\partial}{\partial \tau} \mathcal{W}_{\tau}\left[\bar{\rho}_{\tau}\right]=\frac{1}{2} \int d^{4} x d^{4} y \frac{\delta}{\delta \bar{\rho}_{\tau}^{a}(x)} \chi^{a b}(x, y) \frac{\delta}{\delta \bar{\rho}_{\tau}^{b}(y)} \mathcal{W}_{\tau}\left[\bar{\rho}_{\tau}\right]
$$

This corresponds to the fact, as we mentioned before in Sec.IIIB the correct ordering implies the virtual term $\sigma^{a}(x)$. We obtained in Sec. IIIB the same ordering as in A12 which is determined by the condition that the commutation relation (45) becomes free from the ordering problem. The ordering as shown in (A12) implicitly signifies the presence of the virtual contribution through (A11). In this way, we should consider that our derivation contains the virtual term as well in an implicit way, which has also been mentioned in part in [26].

[1] L. N. Lipatov, Sov. J. Nucl. Phys. 23, 338 (1976) [Yad. Fiz. 23, 642 (1976)].

[2] E. A. Kuraev, L. N. Lipatov and V. S. Fadin, Sov. Phys. JETP 44, 443 (1976) [Zh. Eksp. Teor. Fiz. 71, 840 (1976)].

[3] E. A. Kuraev, L. N. Lipatov and V. S. Fadin, Sov. Phys. JETP 45, 199 (1977) [Zh. Eksp. Teor. Fiz. 72, 377 (1977)].

[4] I. I. Balitsky and L. N. Lipatov, Sov. J. Nucl. Phys. 28, 822 (1978) [Yad. Fiz. 28, 1597 (1978)].

[5] L. V. Gribov, E. M. Levin and M. G. Ryskin, Phys. Rept. 100, 1 (1983).

[6] A. H. Mueller, Nucl. Phys. B 307, 34 (1988).

[7] L. D. McLerran and R. Venugopalan, Phys. Rev. D 49, 2233 (1994) arXiv:hep-ph/9309289.

[8] L. D. McLerran and R. Venugopalan, Phys. Rev. D 49, 3352 (1994) arXiv:hep-ph/9311205.

[9] L. D. McLerran and R. Venugopalan, Phys. Rev. D 50, 2225 (1994) arXiv:hep-ph/9402335.

[10] For reviews on the color glass condensate, see; E. Iancu, A. Leonidov and L. McLerran, arXiv:hep-ph/0202270 E. Iancu and R. Venugopalan, arXiv:hep-ph/0303204

[11] K. Golec-Biernat, L. Motyka and A. M. Stasto, Phys. Rev. D 65, 074037 (2002) arXiv:hep-ph/0110325. 
[12] J. Jalilian-Marian, A. Kovner, A. Leonidov and H. Weigert, Nucl. Phys. B 504, 415 (1997) arXiv:hep-ph/9701284.

[13] J. Jalilian-Marian, A. Kovner, A. Leonidov and H. Weigert, Phys. Rev. D 59, 014014 (1999) arXiv:hep-ph/9706377.

[14] J. Jalilian-Marian, A. Kovner and H. Weigert, Phys. Rev. D 59, 014015 (1999) arXiv:hep-ph/9709432.

[15] A. Kovner, J. G. Milhano and H. Weigert, Phys. Rev. D 62, 114005 (2000) arXiv:hep-ph/0004014.

[16] E. Iancu, A. Leonidov and L. D. McLerran, Nucl. Phys. A 692, 583 (2001) arXiv:hep-ph/0011241.

[17] E. Iancu, A. Leonidov and L. D. McLerran, Phys. Lett. B 510, 133 (2001) arXiv:hep-ph/0102009.

[18] E. Ferreiro, E. Iancu, A. Leonidov and L. McLerran, Nucl. Phys. A 703, 489 (2002) arXiv:hep-ph/0109115.

[19] K. Fukushima, arXiv:hep-ph/0512138 to appear in Nucl. Phys. A.

[20] J. P. Blaizot, E. Iancu and H. Weigert, Nucl. Phys. A 713, 441 (2003) arXiv:hep-ph/0206279.

[21] J. Jalilian-Marian, S. Jeon and R. Venugopalan, Phys. Rev. D 63, 036004 (2001) arXiv:hep-ph/0003070.

[22] P. A. M. Dirac, Lectures in Quantum Mechanics, Belfer Graduate School of Science, Yeshiva University Press, New York, 1964.

[23] Y. Hatta, arXiv:hep-ph/0511287

[24] A. H. Mueller, A. I. Shoshi and S. M. H. Wong, Nucl. Phys. B 715, 440 (2005) arXiv:hep-ph/0501088.

[25] A. Kovner and M. Lublinsky, Phys. Rev. D 71, 085004 (2005) arXiv:hep-ph/0501198.

[26] Y. Hatta, E. Iancu, L. McLerran, A. Stasto and D. N. Triantafyllopoulos, Nucl. Phys. A 764, 423 (2006) arXiv:hep-ph/0504182 .

[27] A. H. Mueller, Nucl. Phys. B 415, 373 (1994).

[28] E. Iancu and A. H. Mueller, Nucl. Phys. A 730, 460 (2004) arXiv:hep-ph/0308315. 